\title{
Nonlinear dynamics in a single mode three-level laser without inversion
}

\author{
A. G. Vladimirov and Paul Mandel \\ Optique Nonlinéaire Théorique, Université Libre de Bruxelles, Campus Plaine Code Postal 231, B-1050 Bruxelles, Belgium \\ S. F. Yelin, ${ }^{1,2,3}$ M. D. Lukin, ${ }^{1,3}$ and M. O. Scully ${ }^{1,3}$ \\ ${ }^{1}$ Department of Physics, Texas A\&M University, College Station, Texas 77843 \\ ${ }^{2}$ Sektion Physik, Ludwig-Maximilians-Universität, D-80333 München, Germany \\ ${ }^{3}$ Max-Planck-Institut für Quantenoptik, D-85748 Garching, Germany
}

(Received 26 August 1997)

\begin{abstract}
We characterize analytically the nature of the lasing solutions of a laser operating without population inversion. We consider models involving three-level media interacting with a strong driving field and a lasing field in the vicinity of the lasing threshold. We assess the influence of different relaxation and pumping schemes on the dynamics of these lasers. We use the atom-field detuning, the cavity detuning, and the linear gain as bifurcation parameters. Depending on their values, the stable lasing solution is shown to be cw or self-pulsing. We show that the optimal lasing operation is achieved if both the driving field frequency and the cavity frequency are out of resonance with the atomic frequencies. Physically, the lasing regimes arise from nonlinearly interacting sidebands induced in the lasing medium by atomic interference.
\end{abstract}

[S1063-651X(98)07201-8]

PACS number(s): 05.45.+b, 42.50.-p, 42.55.-f

\section{INTRODUCTION}

It is by now generally recognized that atomic coherence and interference effects can be used to modify dramatically the response of coherently prepared atomic systems [1]. Amplification and lasing without inversion (LWI) are among the most interesting applications of these effects.

The key mechanism for LWI, proposed theoretically by several authors at the end of the 1980s [2-4] is the cancellation of atomic absorption due to quantum interference. In this case the absorption and emission profiles become nonreciprocal and light amplification is possible even if the population of the upper levels is less than that of the ground state. The interest in inversionless amplification and lasing is stimulated by possible applications, which include the generation of coherent radiation in the UV and $\mathrm{x}$-ray spectral regions, where population inversion is very hard or even impossible to achieve.

The first proposals on LWI were followed by a large number of theoretical publications on the subject [5-7] in which different schemes and different aspects of inversionless gain were studied. Relatively recently, experimental evidence for inversionless amplification and lasing was obtained [8-13]. The experimental and theoretical efforts are presently directed towards the demonstration the inversionless lasing in a frequency up-conversion regime [14].

Up to now the major part of the theoretical literature on the subject of LWI was devoted to the analysis of the linear gain properties of the different amplifiers. Very few papers have dealt with nonlinear dynamical analyses of the lasing regimes [15-18]. The subject of the present paper is to provide an in-depth analysis of the nonlinear dynamical properties of inversionless laser oscillators.

We are motivated by the following considerations. First of all, from a general viewpoint it is clear that inversionless lasers can exhibit dynamical behavior that is very different from that of ordinary laser systems. The coherently prepared active media possesses typically a fairly complicated response that must affect the dynamical behavior of the inversionless laser oscillators.

Secondly, we are specifically interested in regimes of inversionless lasing in which coherent preparation is achieved on slowly decaying, weakly allowed transitions. As was shown recently [14], these regimes are of considerable practical interest when there is a large frequency up-conversion. The linear susceptibility spectrum for such systems often displays amplification in several separate frequency domains, which can lead to generation at several different frequencies simultaneously and thus produce an unstable intensity output. In fact, recent studies $[17,18]$ indicate that in a similar system laser oscillation can occur via a Hopf bifurcation, which can result in self-pulsing intensity. The stability of the various lasing regimes (such as $\mathrm{cw}$ and self-pulsed, for instance) and the possibility to control them are therefore important practical considerations in the up-conversion regime of LWI at least for certain schemes. Similarly, the optimal domain of parameters such as drive field strength and detuning yielding maximal intensity output is often not obvious for such schemes and requires special consideration.

The present paper introduces a general formalism and investigates the dynamics of single-mode inversionless lasers. We start by outlining the formalism applicable to any singlemode laser with a lasing medium consisting of nearly resonant three-level atoms at or near laser threshold. We investigate the character and stability of the emerging solutions.

We first consider the case of zero detunings: a cavity eigenfrequency coincides with the atomic transition frequency coupled by the lasing field and the driving field is resonant with the driven transition. It was shown in [17] that for this scheme both steady and Hopf bifurcations of the trivial solution are possible. We find that the Hopf bifurcation is actually a codimension-two degenerate Hopf bifurca- 
tion that cannot be fully described with the real equations used in [17]. In particular, using the normal form method, we study in detail the solutions emerging from the degenerate Hopf bifurcation. Our analysis shows that even in the case of zero detunings the solution emerging from this bifurcation can lead to either a time-independent or a time-periodic intensity.

We point out that LWI via Hopf bifurcation occurs in the regime where the polarization decay rate of the driven transition is slower than the polarization decay rate of the lasing transition, a regime interesting for LWI with frequency upconversion [14]. It was shown in [14] that the domain of inversionless gain as well as the value of gain itself can be increased by detuning the laser cavity or the driving field from atomic resonance. We find that detuning can indeed substantially increase the domain of LWI oscillation. Furthermore, in the domain that is interesting for the experimental realization of frequency up-conversion, we find that the stability and character of the lasing field can be controlled by properly tuning the laser cavity and driving field. We show that for the general case of nonzero detunings, the trivial solution undergoes a bifurcation leading to $\mathrm{cw}$ laser operation. The emerging solution with time-independent intensity may or may not undergo a secondary Hopf bifurcation for larger values of laser gain.

In the conclusion, we discuss the physical origin of the different LWI regimes described in the present paper.

\section{LASER MODEL AND LINEAR STABILITY ANALYSIS}

\section{A. Basic equations}

We consider the general model of a ring single mode laser consisting of three-level atoms. A lasing field with complex Rabi frequency $\alpha$ couples one of the atomic transitions $(3 \leftrightarrow 1)$. An external driving field with real Rabi frequency $\Omega$ is tuned close to the resonance with another atomic transition $(2 \leftrightarrow 1)$. Driving and lasing fields can interact via the coherence generated on the remaining uncoupled transition $3 \leftrightarrow 2$ (Fig. 1).

The equations describing this model [19-21] can be written in the following form:

$$
\begin{gathered}
\frac{d \alpha}{d t}=-\kappa \alpha+g s x_{31}, \\
\frac{d x_{31}}{d t}=-\left(\gamma_{a}-i \Delta_{a}\right) x_{31}+\alpha\left(1-\rho_{22}-2 \rho_{11}\right)+\Omega x_{32}, \\
\frac{d x_{32}}{d t}=-\left(\gamma_{b}-i \Delta_{a}+i \Delta_{d}\right) x_{32}-\alpha x_{21}-\Omega x_{31}, \\
\frac{d x_{21}}{d t}=-\left(\gamma_{d}+i \Delta_{d}\right) x_{21}-\Omega\left(\rho_{11}-\rho_{22}\right)+\alpha^{*} x_{32}, \\
\frac{d \rho_{11}}{d t}=R_{1}+\Omega\left(x_{21}+x_{21}^{*}\right)+\alpha x_{31}^{*}+\alpha^{*} x_{31}, \\
\frac{d \rho_{22}}{d t}=R_{2}-\Omega\left(x_{21}+x_{21}^{*}\right),
\end{gathered}
$$
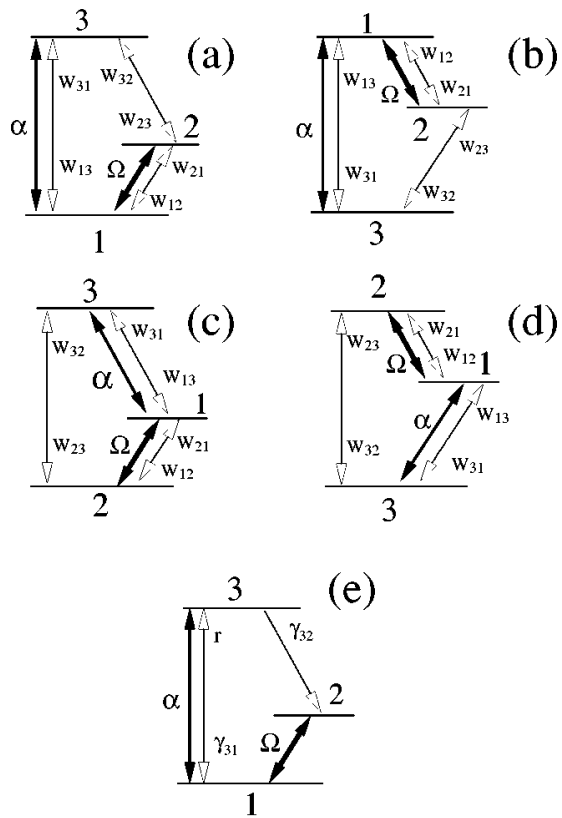

FIG. 1. Three-level LWI schemes. (a) $h(V)$ scheme. (b) $p(\Lambda)$ scheme. (c) Lower-ladder scheme. (d) Upper-ladder scheme. (e) $h$ scheme for frequency up-conversion with driving field applied to the weakly allowed transition $1 \leftrightarrow 2$.

where

$$
\begin{gathered}
\left(\begin{array}{l}
R_{1} \\
R_{2}
\end{array}\right)=\left(\begin{array}{ll}
P & Q \\
R & S
\end{array}\right)\left(\begin{array}{l}
\rho_{11} \\
\rho_{22}
\end{array}\right)+\left(\begin{array}{l}
W_{13} \\
W_{23}
\end{array}\right), \\
P=-\left(W_{21}+W_{31}+W_{13}\right), \quad Q=W_{12}-W_{13}, \\
R=W_{21}-W_{23}, \quad S=-\left(W_{12}+W_{32}+W_{23}\right) .
\end{gathered}
$$

Here the complex variables $x_{31}=i \rho_{31}, x_{32}=\rho_{32}$, and $x_{21}=-i \rho_{21}^{*}$ are proportional to the off-diagonal elements of the density matrix $\left\{\rho_{i k}\right\} . \rho_{11}$ and $\rho_{22}$ are the populations of the levels 1 and 2 . The population of the level 3 is $\rho_{33}=1-\rho_{11}-\rho_{22}$. The coefficient $g$ is defined through

$$
g=2 \pi i N \omega_{c}\left|\mu_{12}\right|^{2} / c_{l} \varepsilon_{l} \hbar,
$$

where $N$ is the number of atoms, $\omega_{c}$ is the cavity eigenfrequency closest to the lasing atomic transition frequency, $\mu_{12}$ is the dipole matrix element excited by the lasing field, $c_{l}$ and $\varepsilon_{l}$ are the speed of light and the dielectric permittivity at the lasing field frequency.

All the parameters in Eqs. (1) are real. These equations describe all possible three-level systems (Fig. 1) interacting with a single mode lasing field and a single frequency driving field. $s=1$ in the case of $h$ or $V$ [Figs. 1(a) and 1(e)] and lower-ladder [Fig. 1(c)] schemes and $s=-1$ in the case of $p$ or $\Lambda$ [Fig. 1(b)] and upper-ladder [Fig. 1(d)] schemes. Cavity and driving field detunings are defined as $\Delta_{a}=\omega_{c}-\omega_{31}$ and $\Delta_{d}=\omega_{d}-\omega_{21}$, respectively, where $\omega_{i j}$ is the atomic frequency of the $i \rightarrow j$ transition and $\omega_{d}$ is the driving field frequency. $\kappa$ is the cavity damping rate. $\left\{W_{i \leftarrow k}\right\}$ in Eq. (2) is the matrix describing incoherent pumping and population relaxation processes. It depends upon the particular choice of three-level system (see Fig. 1) and will be specified later. 

by

Finally, the atomic polarization damping rates are defined

$$
\begin{aligned}
& \gamma_{a}=\gamma_{a}^{n r d}+\left(W_{31}+W_{13}+W_{23}+W_{21}\right) / 2, \\
& \gamma_{b}=\gamma_{b}^{n r d}+\left(W_{32}+W_{23}+W_{13}+W_{12}\right) / 2, \\
& \gamma_{d}=\gamma_{d}^{n r d}+\left(W_{21}+W_{12}+W_{31}+W_{32}\right) / 2,
\end{aligned}
$$

where $\gamma_{a}^{n r d}, \gamma_{b}^{n r d}$, and $\gamma_{d}^{n r d}$ describe the effect of phasedestroying processes. The radiative limit is defined by the conditions

$$
\gamma_{a}^{n r d}=\gamma_{b}^{n r d}=\gamma_{d}^{n r d}=0
$$

The nonlasing steady-state solution of Eqs. (1) corresponding to laser below threshold is given by

$$
\begin{gathered}
\alpha=x_{31}=x_{32}=0, x_{21}=x_{21}^{0}=\frac{\Omega}{\gamma_{d}+i \Delta_{d}}\left(\rho_{22}^{0}-\rho_{11}^{0}\right), \\
\rho_{k k}=\rho_{k k}^{0}, \quad(k=1,2,3) .
\end{gathered}
$$

Here $\rho_{k k}^{0}$ are the atomic populations in the absence of the lasing field $(\alpha=0)$

$$
\begin{gathered}
\rho_{11}^{0}=\left[2 \Omega^{2}\left(W_{13}+W_{23}\right)+\widetilde{\gamma}_{d}\left(Q W_{23}-S W_{13}\right)\right] / D, \\
\rho_{22}^{0}=\left[2 \Omega^{2}\left(W_{13}+W_{23}\right)+\widetilde{\gamma}_{d}\left(R W_{13}-P W_{23}\right)\right] / D, \\
\rho_{33}^{0}=1-\rho_{22}^{0}-\rho_{33}^{0},
\end{gathered}
$$

where

$$
\begin{gathered}
D=-2 \Omega^{2}(P+Q+R+S)+\widetilde{\gamma}_{d}(P S-Q R), \\
\widetilde{\gamma}_{d}=\gamma_{d}+\Delta_{d}^{2} / \gamma_{d} .
\end{gathered}
$$

In the following, the population differences defined by

$$
n_{i j}=\rho_{i i}^{0}-\rho_{j j}^{0}
$$

will be used.

\section{B. Linear stability analysis}

We now proceed with the linear stability analysis of the trivial solution (6). The system (1) is linearized about the solutions (6). The resulting Jacobian matrix has a blockdiagonal form with two blocks $L_{1}$ and $L_{2}$ (see Appendix). The stability of the steady-state solution (6) depends on the eigenvalues of the complex matrix $L_{1}$. The characteristic equation is given by

$$
\begin{gathered}
P_{1}(\lambda) P_{2}(\lambda)=0 \\
P_{1}(\lambda)=\sum_{n=0}^{3} \lambda^{n} A_{n}, \quad P_{2}(\lambda)=\sum_{n=0}^{3} \lambda^{n} A_{n}^{*},
\end{gathered}
$$

where $A_{n}^{*}$ is the complex conjugate of $A_{n}$, and

$$
\begin{gathered}
A_{3}=1, \\
A_{2}=\kappa+\Gamma_{a}+\Gamma_{b}, \\
A_{1}=\kappa\left(\Gamma_{a}+\Gamma_{b}\right)+\Gamma_{a} \Gamma_{b}+\Omega^{2}+g s n_{13}, \\
A_{0}=\kappa\left(\Omega^{2}+\Gamma_{a} \Gamma_{b}\right)+g s\left(\frac{\Omega^{2}}{\Gamma_{d}} n_{21}+\Gamma_{b} n_{13}\right) .
\end{gathered}
$$

Here $\quad \Gamma_{a}=\gamma_{a}-i \Delta_{a}, \quad \Gamma_{b}=\gamma_{b}-i \Delta_{a}+i \Delta_{d}, \quad$ and $\quad \Gamma_{d}=\gamma_{d}$ $+i \Delta_{d}$. In what follows we label the characteristic roots $\left\{\lambda_{i}\right\}$ such that $\operatorname{Re} \lambda_{1} \geqslant \operatorname{Re} \lambda_{2} \cdots \geqslant \operatorname{Re} \lambda_{6}$. Thus the trivial solution (6) becomes unstable when $\operatorname{Re} \lambda_{1}=0$. We will in the following use $\Delta_{a}, \Delta_{d}$, and $g$ as bifurcation parameters.

\section{The resonant case}

Let us first consider the case of zero detunings $\left(\Delta_{a}=\Delta_{d}=0\right)$ so that $P_{1}(\lambda)=P_{2}(\lambda)$ are real. For this case, bifurcations of the nonlasing solution of the real Eqs. (1) were described in detail [17]. The steady bifurcation point of Eq. (6) is given by

$$
\Delta_{a}=\Delta_{d}=0, \quad g=g_{0}=-\frac{\kappa \gamma_{d}\left(\Omega^{2}+\gamma_{a} \gamma_{b}\right)}{s\left(\Omega^{2} n_{21}+n_{13} \gamma_{b} \gamma_{d}\right)} .
$$

At this point, the characteristic equation (10) has two zero eigenvalues. The solution (6) is stable (unstable) for $g<g_{0}$ $\left(g>g_{0}\right)$. This bifurcation leads to a regime with stationary intensity appearing at the lasing transition frequency. Since $g_{0}$ in Eq. (12) should be positive, a necessary condition for the steady-state bifurcation is

$$
s\left(\Omega^{2} n_{21}+n_{13} \gamma_{b} \gamma_{d}\right)<0
$$

The inversionless condition implies $n_{13}>0$ for the $h$ and lower-ladder schemes and $n_{31}=-n_{13}>0$ for the $p$ and upper-ladder schemes. Therefore, the necessary condition for the steady-state bifurcation in a perfectly tuned laser is $n_{21}<0\left(n_{12}<0\right)$ for $s=1(s=-1)$. It is easy to check that this also means that in the absence of the driving field the population of level 1 should be greater (smaller) than that of the level 2 for $s=1(s=-1)[21,17]$.

It was shown in [17] that when both detuning parameters are equal to zero the nonlasing solution can also exhibit a Hopf bifurcation. This Hopf bifurcation is defined by

$\Delta_{a}=\Delta_{d}=0, \quad g=g_{0}=\frac{\gamma_{d}\left(\gamma_{a}+\gamma_{b}\right)\left[\Omega^{2}+\left(\gamma_{a}+\kappa\right)\left(\gamma_{b}+\kappa\right)\right]}{s\left[\Omega^{2} n_{21}-n_{13} \gamma_{d}\left(\kappa+\gamma_{a}\right)\right]}$

In our analysis the variables $\alpha, x_{31}, x_{32}$, and $x_{21}$ are complex, so that we have a codimension-two degenerate Hopf bifurcation instead of the generic codimension-one bifurcation, which takes place in the real laser equations used in [17]. This means that the characteristic equation has a pair of pure imaginary roots $\left(i \omega_{0},-i \omega_{0}\right)$, which is doubly degenerate: both $P_{1}(\lambda)$ and $P_{2}(\lambda)$ have the same pair of roots $\left(i \omega_{0},-i \omega_{0}\right)$, where $\omega_{0}$ is defined by 


$$
\omega_{0}^{2}=\Omega^{2}+\frac{n_{21} \Omega^{2}\left[\kappa\left(\gamma_{a}+\gamma_{b}\right)+\gamma_{a} \gamma_{b}\right]+n_{13} \gamma_{d}\left[\Omega^{2}\left(\gamma_{a}+\gamma_{b}\right)+\gamma_{b}^{2}\left(\kappa+\gamma_{a}\right)\right]}{\Omega^{2} n_{21}-n_{13} \gamma_{d}\left(\kappa+\gamma_{a}\right)} .
$$

The degenerate Hopf bifurcation set (13) is the intersection of two codimension-one surfaces in parameter space. Each of these surfaces corresponds to a generic Hopf bifurcation and is associated with a sideband instability leading to a timeperiodic solution of Eq. (1), which, however, corresponds to a cw laser operation. The degenerate Hopf bifurcation takes place when instabilities on both sidebands occur simultaneously.

For positive values of $g_{0}$ in Eq. (13) the nonlasing solution (6) is unstable for $g>g_{0}$. If $g_{0}$ defined by Eq. (13) is negative then the Hopf bifurcation is impossible. Hence, the necessary condition for the degenerate Hopf bifurcation (13) is given by

$$
D_{0} \equiv s\left[\Omega^{2} n_{21}-n_{13} \gamma_{d}\left(\kappa+\gamma_{a}\right)\right]>0 .
$$

For the $h$ and lower-ladder schemes ( $p$ and upper-ladder schemes) shown in Figs. 1(a), 1(c), and 1(e) [Figs. 1(b) and $1(\mathrm{~d})]$ this condition takes the form $\Omega^{2} n_{21}-n_{13} \gamma_{d}\left(\kappa+\gamma_{a}\right)$ $>0\left[\Omega^{2} n_{12}-n_{31} \gamma_{d}\left(\kappa+\gamma_{a}\right)<0\right]$ and, hence, the degenerate Hopf bifurcation (13) is possible only for $n_{21}>0\left(n_{12}>0\right)$ $[21,17]$. Thus, the Hopf bifurcation requires a positive (negative) population difference between the levels 2 and 1 for $s=1 \quad(s=-1)$ and it is incompatible with the steady-state bifurcation (15) [17]. Note that the threshold value $g_{0}$ defined by Eq. (13) does not vanish for $\kappa \rightarrow 0$ as it does in the case of the steady-state bifurcation (12). As shown below, this results from the finite amount of atomic dispersion required to compensate cavity resonances and to allow for sidebands to oscillate even in the limit of a perfect cavity $(\kappa \rightarrow 0)$.

\section{The case of small detunings}

We first seek a steady-state bifurcation that is defined by $\lambda_{1}=0$. This means $A_{0}=0$ in Eq. (11), and therefore also $\lambda_{2}=0$. In this case a cw field emerges in resonance with one of the cavity eigenfrequencies.

If we redefine $\lambda \equiv \tilde{\lambda}-i \Delta$ with arbitrary $\Delta$, the characterisitc Eq. (10) becomes

$$
P_{1}(\tilde{\lambda}-i \Delta) P_{2}(\tilde{\lambda}+i \Delta)=0
$$

In order to find a solution $\widetilde{\lambda}=0$ we have to solve

$$
\widetilde{A_{0}}=i \Delta^{3}-A_{2} \Delta^{2}-i A_{1} \Delta+A_{0}=0
$$

for $\Delta$ and the coupling $g$. We find with $\tilde{\lambda_{1,2}}=0$ a simple Hopf bifurcation $\lambda_{1,2}= \pm i \Delta$, leading to a $\mathrm{cw}$ laser output with detuning $\Delta$ relative to the cavity resonance. It becomes clear that the case $\lambda_{1,2}=0$ is just a special solution with $\Delta=0$. In the general case, the analysis of Eq. (16) requires the explicit solution of an irreducible third order polynomial. Therefore, we confine our analytical consideration to the limit of small cavity and drive detunings. In this limit, the characteristic equation (10) can be linearized in $\Delta_{d}$ and $\Delta_{a}$.
At order zero in $\Delta_{a}$ and $\Delta_{d}$, the instability point is given by Eq. (13). We know that at the bifurcation point $\lambda$ has to be purely imaginary. Therefore, only the $\Delta_{a}$ - or $\Delta_{d}$ - dependent part of $\lambda$ can contribute to its real part. Solving $\operatorname{Re} \lambda=0$, we determine the first order correction to the critical value of the coupling constant $g$. Since there are two solutions of the characteristic equation in the resonant case, $\lambda_{1,2}= \pm i \omega_{0}$, there are two solutions for the linear part of $g$

$$
\begin{gathered}
g=g_{0} \pm \Delta g \\
\Delta g=\frac{1}{\omega_{0} D_{0}}\left\{\Delta_{a} \gamma_{d}\left[\left(\Omega^{2}+\gamma_{b} \gamma_{a}\right)\left(\gamma_{a}+\gamma_{b}+\kappa\right)-\kappa \omega_{0}^{2}\right]\right. \\
-\Delta_{d}\left[( \gamma _ { a } + \gamma _ { b } + \kappa ) \left(\Omega^{2}\left(\gamma_{b}+\gamma_{d}-\kappa\right)+\left(\gamma_{a}+\kappa\right)\right.\right. \\
\left.\left.\left.\times\left(\omega_{0}^{2}+\gamma_{b}^{2}+\gamma_{b} \gamma_{d}\right)\right)-\omega_{0}^{2} \gamma_{d}\left(\gamma_{a}+\kappa\right)\right]\right\}
\end{gathered}
$$

where $g_{0}, \omega_{0}$, and $D_{0}$ are defined by Eqs. (13), (14), and (15), respectively. Each of these two solutions corresponds to a simple Hopf bifurcation. Thus for nonzero detunings the degenerate Hopf bifurcation obtained on resonance splits into two generic Hopf bifurcations. For small $\Delta_{a}$ and $\Delta_{d}$ the first of these two bifurcations occurs for $g=g_{0}-|\Delta g|<g_{0}$ whereas the second one occurs for $g=g_{0}+|\Delta g|>g_{0}$. Thus the instability threshold $g=g_{0}-|\Delta g|$ is lowered in a slightly detuned laser. The codimension-two degenerate Hopf bifurcation takes place when both the Hopf bifurcations take place simultaneously. This condition can be satisfied not only for $\Delta_{a}=\Delta_{d}=0$, but also in the case of small detunings when $\Delta g=0$ in Eq. (18). This gives a relation between $\Delta_{a}$ and $\Delta_{d}$, which together with $g=g_{0}$ determines the linear approximation to a codimension-two bifurcation set corresponding to a degenerate Hopf bifurcation. Note that for $\kappa$ $\rightarrow 0$ and $\gamma_{a}+\gamma_{b}>\gamma_{d}$ the detuning parameters $\Delta_{a}$ and $\Delta_{d}$ should have the same sign in order to satisfy the degenerate Hopf bifurcation condition $\Delta g=0$.

\section{DEGENERATE HOPF BIFURCATION}

Bifurcation phenomena near the degenerate Hopf bifurcation point (13) can be described using the normal form method [22-24]. The truncated normal form equations for this bifurcation are, in general, given by [25]

$$
\begin{aligned}
& \dot{z}_{+}=z_{+}\left(\beta+\delta-C_{1}\left|z_{+}\right|^{2}-C_{2}\left|z_{-}\right|^{2}\right), \\
& \dot{z}_{-}=z_{-}\left(\beta-\delta-C_{1}\left|z_{-}\right|^{2}-C_{2}\left|z_{+}\right|^{2}\right) .
\end{aligned}
$$

Here the complex variables $z_{+}$and $z_{-}$are the slowly varying in time envelopes of the two sidebands (see Appendix). The complex parameters $\beta$ and $\delta$ describe the small deviation of the parameters $\Delta_{a}, \Delta_{d}$, and $g$ from the degenerate Hopf bifurcation point (13). The outline of the procedure used to derive Eqs. (19) and the explicit form of the parameters $\beta$ and $\delta$ are given in Appendix A. 

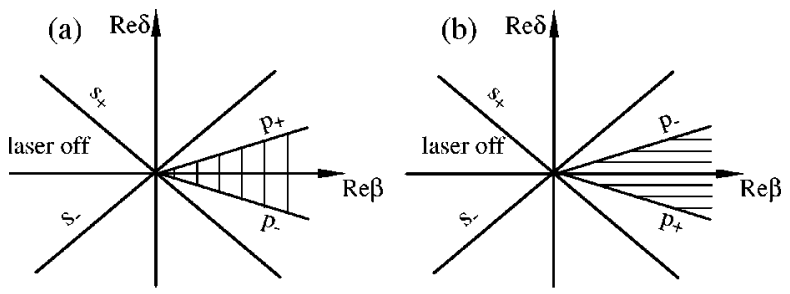

FIG. 2. Bifurcation diagrams for the normal form equations (19). $\operatorname{Re} C_{1}>0$ and $\operatorname{Re} C_{2}>0$ for both diagrams. The time-periodic solution $S_{+}\left(S_{-}\right)$of Eq. (19) corresponds to cw laser operation and exists above (below) the line $s_{+}\left(s_{-}\right)$. It is stable above (below) the line $p_{+}\left(p_{-}\right)$. (a) $\operatorname{Re} C_{1}>\operatorname{Re} C_{2}$. The stable quasiperiodic solution $P$ of Eq. (19) exists in the vertically dashed wedge between the lines $p_{+}$and $p_{-}$. This solution corresponds to a self-pulsed laser output. (b) $\operatorname{Re} C_{1}<\operatorname{Re} C_{2}$. In the horizontally dashed wedge the solution $P$ is unstable. Two solutions with cw laser output $\left(S_{+}\right.$and $S_{-}$) are bistable in this wedge.

The codimension-two bifurcation point of Eqs. (19) $\operatorname{Re} \beta=\operatorname{Re} \delta=0$ corresponds to the degenerate Hopf bifurcation of Eqs. (1) and it lies on the intersection of the two Hopf bifurcation lines $\operatorname{Re} \beta=\operatorname{Re} \delta$ and $\operatorname{Re} \beta=-\operatorname{Re} \delta$. In the particular case $\Delta_{a}=\Delta_{d}=0$, we have $\operatorname{Im} \beta=\operatorname{Re} \delta=0$ in Eq. (19). The type of the bifurcation diagram associated with Eqs. (19) depends on the real parts of the coefficients $C_{1}$ and $C_{2}$, which can be calculated for $\Delta_{a}=\Delta_{d}=0$. Since for the schemes under consideration we have not found situations in which either $\operatorname{Re} C_{1}<0$ or $\operatorname{Re}\left(C_{1}+C_{2}\right)<0$, we consider only the case where both $\operatorname{Re} C_{1}$ and $\operatorname{Re}\left(C_{1}+C_{2}\right)$ are positive. Then all the branches of solutions bifurcating from the non-lasing solution (6) are supercritical (i.e., they exist above the instability threshold) and the bifurcation diagram for Eqs. (19) depends on the sign of the quantity $\operatorname{Re}\left(C_{1}-C_{2}\right)$, as shown in Fig. 2.

The steady-state $z_{+}=z_{-}=0$ of Eq. (19) corresponds to the nonlasing solution (6) and it is stable below the lasing threshold $(\operatorname{Re} \beta<0,|\operatorname{Re} \delta|<|\operatorname{Re} \beta|)$. Above this threshold and for sufficiently small detunings

$$
\operatorname{Re} \beta>0, \quad\left|\operatorname{Re} \delta / \operatorname{Re}\left(C_{1}-C_{2}\right)\right|<\operatorname{Re} \beta / \operatorname{Re}\left(C_{1}+C_{2}\right),
$$

there exists a stable quasiperiodic solution $P$ of Eq. (19) with $\left|z_{1}\right|^{2}=\operatorname{Re} \varepsilon / \operatorname{Re}\left(C_{1}+C_{2}\right)+\operatorname{Re} \delta / \operatorname{Re}\left(C_{1}-C_{2}\right)$ and $\left|z_{-}\right|^{2}$ $=\operatorname{Re} \beta / \operatorname{Re}\left(C_{1}+C_{2}\right)-\operatorname{Re} \delta / \operatorname{Re}\left(C_{1}-C_{2}\right)$. This solution corresponds to a laser output with the intensity oscillating in time at a frequency close to the frequency $\omega_{0}$ defined by Eq. (14). There are also two periodic solutions of Eq. (19) that correspond to laser operation with stationary intensity: $S_{+}$ $\left[\left|z_{+}\right|^{2}=\operatorname{Re}(\beta+\delta) /\left(\operatorname{Re} C_{1}\right)^{2},\left|z_{-}\right|^{2}=0\right]$ and $S_{-}\left[\left|z_{+}\right|^{2}=0\right.$, $\left.\left|z_{-}\right|^{2}=\operatorname{Re}(\beta-\delta) /\left(\operatorname{Re} C_{1}\right)^{2}\right]$. The solution $S_{+}\left(S_{-}\right)$exists for $\operatorname{Re} \beta>-\operatorname{Re} \delta(\operatorname{Re} \beta>\operatorname{Re} \delta)$ and it corresponds to the cw laser output with a frequency shifted from the resonance frequency of the lasing transition by an amount approximately equal to $\omega_{0}\left(-\omega_{0}\right)$. Note that the solutions $S_{+}$and $S_{-}$do not exist in the real equations studied in [17].

If the self-saturation coefficient $\operatorname{Re} C_{1}$ in Eq. (19) is greater than the cross saturation coefficient $\operatorname{Re} C_{2}$, then the quasiperiodic solution $P$ is stable. This means that the two sidebands lock in to produce a regime with time-periodic intensity. The solution $S_{+}\left(S_{-}\right)$corresponding to stationary laser intensity is stable for $\operatorname{Re} \delta>0(\operatorname{Re} \delta<0)$ only outside the domain (20) where the stable solution $P$ exists. The solution $P$ bifurcates directly from the nonlasing solution only in the case $\operatorname{Re} \delta=0$. If $\operatorname{Re} \delta \neq 0$ but small enough, the solution $S_{+}$(or $S_{-}$) appears first. Increasing $g$, it undergoes a secondary bifurcation giving rise to the solution $P$ with timeperiodic intensity. For negative values of $\operatorname{Re}\left(C_{1}-C_{2}\right)$ the cross saturation for the sidebands is stronger than the selfsaturation. Therefore the laser cannot operate on two sidebands simultaneously and the solution $P$ is unstable. In this case we have bistability between $S_{+}$and $S_{-}$in the parameter region where both these solutions coexist with the unstable solution $P$. Outside of this region but still above the threshold there is only one stable solution with nonzero timeindependent laser intensity $\left(S_{+}\right.$for $\delta>0$ and $S_{-}$for $\left.\delta<0\right)$.

Thus, the solutions with periodic laser intensity are associated with the degenerate Hopf bifurcation (13). They can exist only if the gain of both sidebands is sufficiently large, so that both of them are involved in the radiation generation. In particular, the sidebands appear simultaneously at the degenerate Hopf bifurcation point. Therefore, the solution $P$ always exists near this point. However, even if it exists it is unstable if $\operatorname{Re}\left(C_{1}-C_{2}\right)>0$. The instability of the solution with time-periodic intensity results from nonsymmetric perturbations $\delta z_{+} \neq \delta z_{-}$of the variables of Eqs. (19). This type of phase instability does not appear in [17] where Eqs. (1) are studied with all variables real, so that one always has $z_{+}=z_{-}$in Eq. (19).

In order to check whether there exist any parameter values for which a stable solution with time-periodic laser intensity can be found near the degenerate Hopf bifurcation point (13) we need to evaluate explicitly the expression $\operatorname{Re}\left(C_{1}-C_{2}\right)$. We have calculated the expressions for the coefficients $C_{1}$ and $C_{2}$ analytically with the help of a symbolic algebra software. Since they are extremely cumbersome, we focus on specific cases and present only numerical results obtained using the analytical expressions for $C_{1,2}$.

In what follows we first consider the model used in [17]. We show that for this model there exists a parameter domain in which the solution with periodic intensity is unstable and above the Hopf bifurcation the laser exhibits only cw regimes. A second case considered corresponds to the model used in [14], which was proposed for the realization of LWI in a frequency up-conversion regime.

\section{ANALYSIS OF SPECIFIC MODELS OF THE LASING MEDIUM}

\section{A. Models of three-level medium}

We now specify the particular pumping and relaxation models, and determine the domain of lasing and the stability of self-pulsed solutions. First, we would like here to make a connection with the results of Ref. [17] and, therefore, use the same model in which it is assumed that detailed balance holds. In this case the matrix $\left\{W_{i \leftarrow j}\right\}$ is defined by [7,19-21]

$$
\begin{aligned}
& W_{12}=N_{1} / T_{21}, \quad W_{21}=N_{2} / T_{21}, \quad W_{13}=N_{1} / T_{31}, \\
& W_{31}=N_{3} / T_{31}, \quad W_{23}=N_{2} / T_{32}, \quad W_{32}=N_{3} / T_{32},
\end{aligned}
$$


where $N_{1}, N_{2}$, and $N_{3}=1-N_{1}-N_{2}$, are the populations of the levels 1,2 , and 3 in the absence of the lasing and the driving fields and $T_{i k}$ are the longitudinal relaxation times.

Substituting Eq. (21) into Eq. (2) we get

$$
\begin{gathered}
R_{1}=-\left(\frac{N_{2}}{T_{21}}+\frac{N_{1}+N_{3}}{T_{31}}\right) \rho_{11}+N_{1}\left(\frac{1}{T_{21}}-\frac{1}{T_{31}}\right) \rho_{22}+\frac{N_{1}}{T_{31}}, \\
R_{2}=N_{2}\left(\frac{1}{T_{21}}-\frac{1}{T_{32}}\right) \rho_{11}-\left(\frac{N_{1}}{T_{21}}+\frac{N_{2}+N_{3}}{T_{32}}\right) \rho_{22}+\frac{N_{2}}{T_{32}} .
\end{gathered}
$$

The steady-state populations and polarization of the driven transition corresponding to the trivial solution $\alpha=0$ follow directly from Eqs. (6) and (7).

The second specific model used here corresponds to the scheme described in [14] for inversionless frequency upconversion. It is similar to the usual $h$ (or $V$ ) system with $s=+1$ in Eq. (1), but with the driving field applied to a weakly allowed transition between the levels 1 and 2 [see Fig. 1(e)]. In this case $\left\{W_{i \leftarrow k}\right\}$ is given by

$$
\begin{aligned}
& W_{12}=0, \quad W_{21}=0, \quad W_{13}=\gamma_{31}, \\
& W_{31}=r, \quad W_{23}=\gamma_{32}, \quad W_{32}=0 . .
\end{aligned}
$$

In order to distinguish easily between the two groups of models, we adopt the follwoing convention. For the four schemes of Figs. 1(a)-1(d), we use decay times $T_{i j}$ while for the scheme of Fig. 1(e), we use decay rates $\gamma_{i j}$. Therefore, we have

$$
\begin{gathered}
R_{1}=-\left(\gamma_{31}+r\right) \rho_{11}-\gamma_{31} \rho_{22}+\gamma_{31}, \\
R_{2}=-\gamma_{32} \rho_{11}-\gamma_{32} \rho_{22}+\gamma_{32} .
\end{gathered}
$$

Since the relaxation matrix (23) is not a particular case of Eq. (21), the scheme shown in Fig. 1(e) requires separate consideration. The steady-state populations (7) for this scheme are given by

$$
\begin{gathered}
\rho_{11}^{0}=2 \Omega^{2}\left(\gamma_{32}+\gamma_{31}\right) / D, \\
\rho_{22}^{0}=\left[2 \Omega^{2}\left(\gamma_{32}+\gamma_{31}\right)+r \gamma_{32} \tilde{\gamma}_{d}\right] / D, \\
\rho_{33}^{0}=2 \Omega^{2} r / D,
\end{gathered}
$$

where

$$
D=r \gamma_{32} \tilde{\gamma}_{d}+2 \Omega^{2}\left[r+2\left(\gamma_{32}+\gamma_{31}\right)\right] .
$$

Here $\widetilde{\gamma}_{d}$ is defined by Eq. (8). It is easy to check that there is no inversion for $\alpha=0$ in this scheme $\left(\rho_{11}^{0}-\rho_{33}^{0}>0\right)$ if the incoherent pump parameter does not exceed the sum of the population relaxation rates $r<\gamma_{32}+\gamma_{31}$ [14]. It follows from Eq. (25) that $n_{21}$ is always positive. Hence, the instability at line center (12) is impossible for this scheme if the driving field is in exact resonance with the atomic transition. The bifurcation conditions can now be easily calculated from the general formulas of Sec. II.

Stable solution with time-periodic lasing intensity exists near the degenerate Hopf bifurcation (13) only if $\operatorname{Re} C_{1}>\operatorname{Re} C_{2}$ in Eq. (19). Using the procedure outlined in the Appendix we have calculated the coefficients $C_{1}$ and $C_{2}$. These coefficients have been evaluated numerically and the
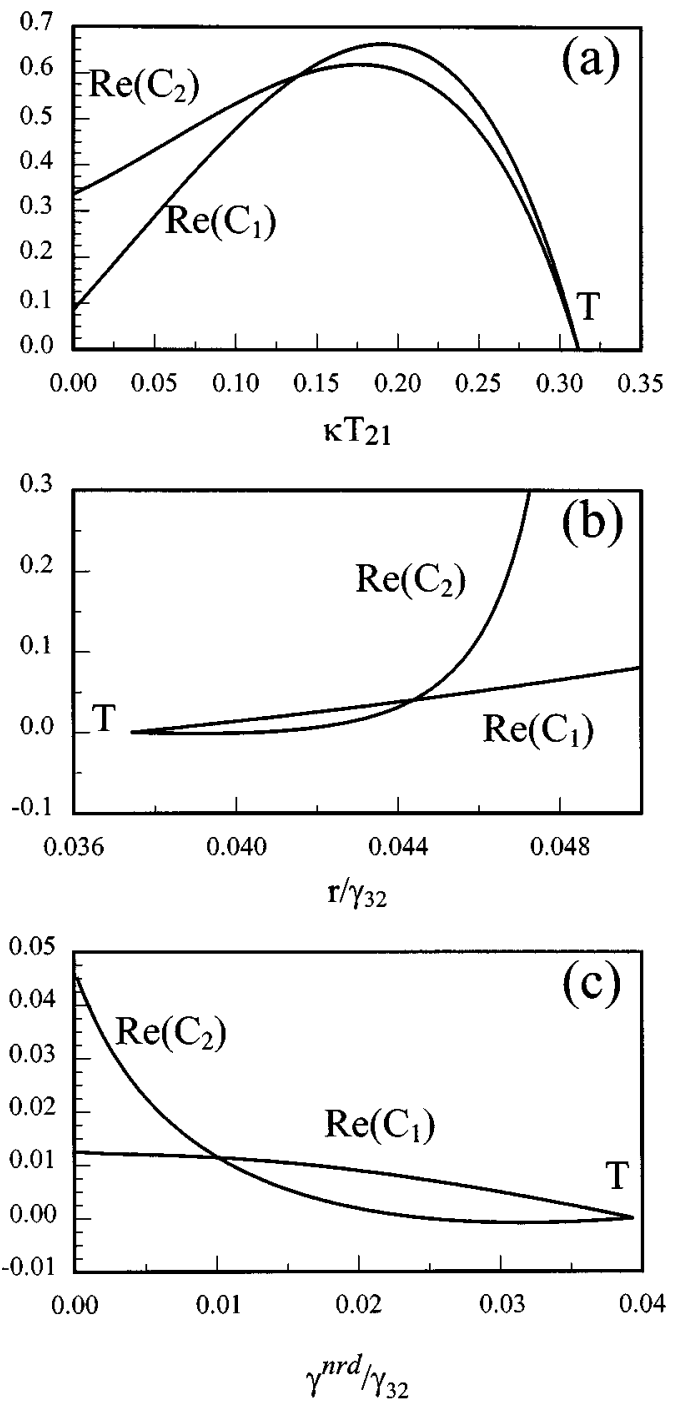

FIG. 3. Real parts of the normal form coefficients $C_{1}$ and $C_{2}$ evaluated at the degenerate Hopf bifurcation point (13). If $\operatorname{Re} C_{1}>\operatorname{Re} C_{2}\left(\operatorname{Re} C_{1}<\operatorname{Re} C_{2}\right)$, a stable (unstable) solution with time-periodic laser output exists near the Hopf bifurcation. The point $T$ indicates the LWI domain boundary. (a) Dependence of $\operatorname{Re} C_{1}$ and $\operatorname{Re} C_{2}$ on the cavity decay rate: $\gamma_{a}^{n r d}=\gamma_{b}^{n r d}=\gamma_{p}^{n r d}=0$, $s=1, T_{31}=10 T_{21}, T_{32}=100 T_{21}, N_{1}=0.22, N_{2}=0.6, \Omega=1 / T_{21}$. (b) Dependence of $\operatorname{Re} C_{1}$ and $\operatorname{Re} C_{2}$ on the incoherent pumping rate for the scheme shown in Fig. 1(e): $\gamma_{a}^{n r d}=\gamma_{b}^{n r d}=\gamma_{p}^{n r d}=0, \kappa$ $=(1 / 3) 10^{-2} \gamma_{32}, \gamma_{31}=(2 / 3) \gamma_{32}, \Omega=10 \gamma_{32} / 3$. (c) Dependence of $\operatorname{Re} C_{1}$ and $\operatorname{Re} C_{2}$ on the phase-destroying rate: $\gamma_{a}^{n r d}=\gamma_{b}^{n r d}$ $=\gamma_{p}^{n r d}=\gamma^{n r d}, \kappa=10^{-2} \gamma_{32}, \gamma_{31}=10^{-1} \gamma_{32}, r=\gamma_{32}, \Omega=10^{2} \gamma_{32}$.

results are shown in Figs. 3(a)-3(c). Figure 3(a) was calculated in the radiative limit (5) with the help of the relaxation matrix defined by Eq. (21). It represents the dependence of $\operatorname{Re} C_{1}$ and $\operatorname{Re} C_{2}$ on the cavity damping rate $\kappa$. In this figure the solution with time-periodic laser intensity is stable only for sufficiently large $\kappa$. On the other hand, if the cavity damping rate is small enough, the self-pulsed solution is unstable and the laser exhibits only a cw regime. Hence, for certain parameter values the Hopf bifurcation described in [17] results in a cw laser operation instead of self-pulsing. The other two graphs in Fig. 3 correspond to the scheme shown in Fig. 1(e). Figure 3(b) was calculated in the radiative limit and represents the dependence of the real parts of 

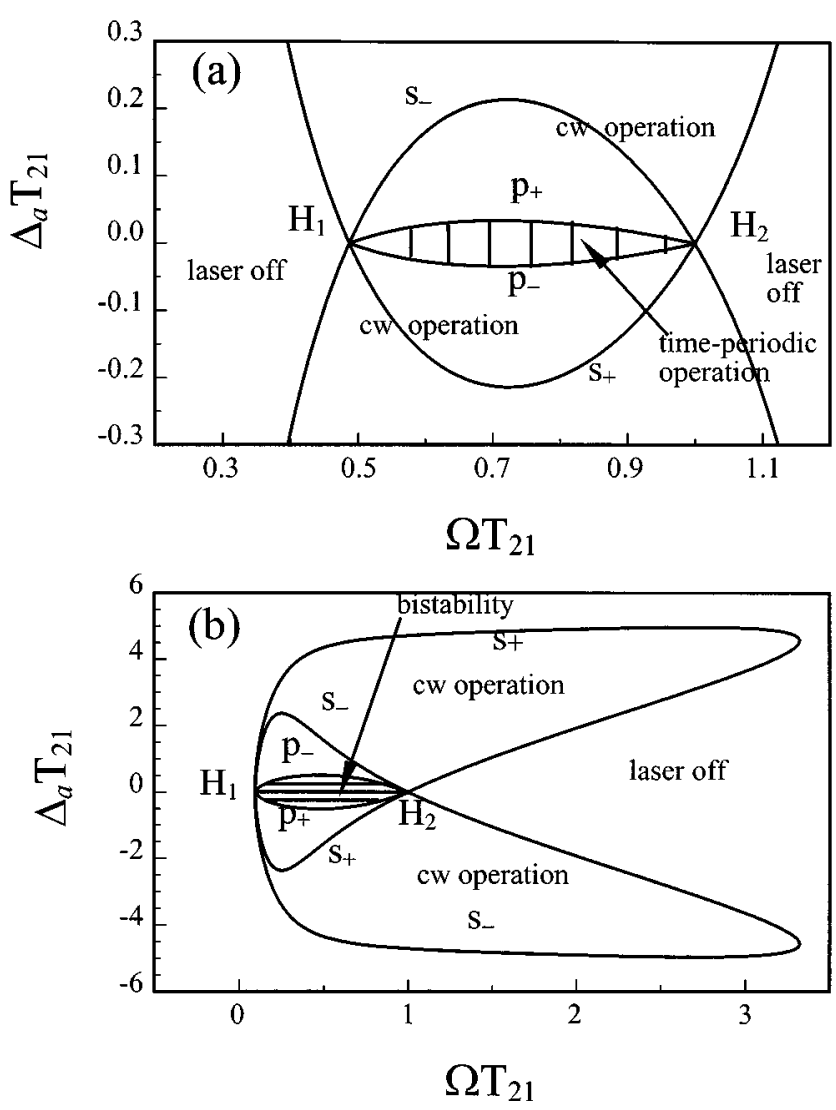

FIG. 4. Bifurcation diagram for Eqs. (1)-(5) and (22). The curves $s_{+}$and $s_{-}$indicate the bifurcations of the nonlasing solution leading to the solutions with cw laser output. The curves $p_{+}$and $p_{-}$ correspond to the bifurcation leading to the solution $P$ with selfpulsed output. (a) Stable solution $P$ exists in the dashed area. $s=-1, \kappa=0.15 / T_{21}, g=53.3416 / T_{21}^{2}, T_{31}=10 T_{21}, T_{32}=100 T_{21}$, $N_{1}=0.38, N_{2}=0.1$. (b) The solution $P$ exists but is unstable in the dashed area where two solutions with $\mathrm{cw}$ output are bistable. $s=-1, \kappa=0.015 / T_{21}, g=8.4157 / T_{21}^{2}, T_{31}=10 T_{21}, T_{32}=100 T_{21}$, $N_{1}=0.42, N_{2}=0.1$.

the normal form coefficients on the incoherent pumping rate $r$. In Fig. 3(b) the solution with time-periodic intensity is stable only for sufficiently small values of $r$. Figure 3(c) corresponds to the case $\gamma_{a}^{n r d}=\gamma_{b}^{n r d}=\gamma_{d}^{n r d}=\gamma^{n r d}$, where $\gamma^{n r d}$ is the contribution of the phase destroying processes to the decay rates. Here we also have either stable or unstable selfpulsed solution. Note that in all three graphs of Fig. 3 the parameter region where the solution with time-periodic laser intensity is stable lies near the LWI domain boundary labelled $T$.

\section{B. Bifurcation diagrams}

To verify the predictions of normal form analysis we have studied the bifurcations of Eqs. (1) numerically. The results of our calculations appear to be in good agreement with those obtained by the normal form method. In Fig. 4 bifurcation curves of Eqs. (1)-(5) and (22) in the $\left(\Omega, \Delta_{a}\right)$ plane are shown. In the case of finite cavity decay rate $(\kappa>0)$ LWI can exist only in a finite range of the driving field intensities. In particular, solving the last equation in (13) for $\Omega^{2}$ we get two positive solutions determining LWI bound-
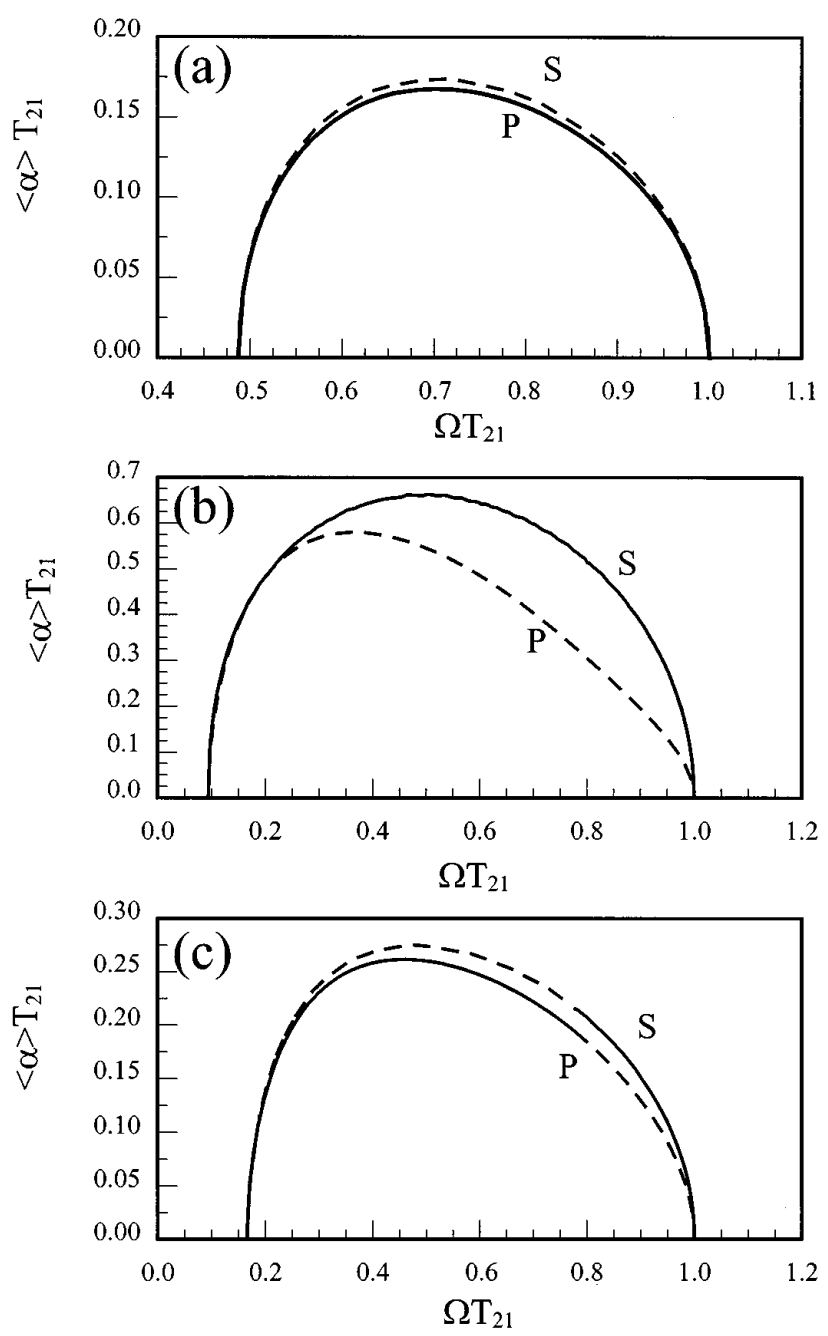

FIG. 5. Branches of the solutions of Eqs. (1)-(5) and (22) with self-pulsed and cw laser output for $\Delta_{a}=\Delta_{d}=0$. Solutions corresponding to $\mathrm{cw}$ (time-periodic) laser intensity are labeled $S(P)$. Stable (unstable) solutions are indicated by a solid (dashed) line. (a) The parameter values are the same as in Fig. 4(a). (b) The parameters are the same as in Fig. 4(b). (c) $s=-1, \kappa=0.15 / T_{21}$, $g=11.7228 / T_{21}^{2}, T_{31}=10 T_{21}, T_{32}=100 T_{21}, N_{1}=0.42, N_{2}=0.1$, $\Omega=1 / T_{21}$.

aries for a laser with $\Delta_{a}=\Delta_{d}=0$. These two solutions correspond to the degenerate Hopf bifurcation points labeled by $H_{1}$ and $H_{2}$ in Fig. 4. In Fig. 4(b) we have $\operatorname{Re}\left(C_{1}-C_{2}\right)<0$ and, hence, the solution $P$ corresponding to time-periodic laser output is unstable. The solution $S_{+}\left(S_{-}\right)$corresponding to cw laser output exists inside the closed curve $s_{+}\left(s_{-}\right)$and it is stable above (below) the curve $p_{+}\left(p_{-}\right)$. The bistability domain for the cw solutions $\left(S_{+}\right.$and $\left.S_{-}\right)$is dashed. It lies between the curves $p_{+}$and $p_{-}$where the unstable solution $P$ exists. Figure 4(a) is similar to Fig. 4(b) but represents only the region of small cavity detunings. Unlike Fig. 4(a), we have $\operatorname{Re}\left(C_{1}-C_{2}\right)>0$ for the codimension-two points $H_{1}$ and $\mathrm{H}_{2}$ shown in Fig. 4(b). In this case we have a stable solution with time-periodic laser output that exists in the dashed area between the curves $p_{+}$and $p_{-}$. The solution $S_{+}\left(S_{-}\right)$with stationary laser intensity exists above the curve $s_{+}\left(s_{-}\right)$and it is stable above (below) the curve $p_{+}\left(p_{-}\right)$. Figure 5 represents the dependence of the laser field intensity on the driving field amplitude $\Omega$ for the case when $\Delta_{a}=\Delta_{d}=0$. The 


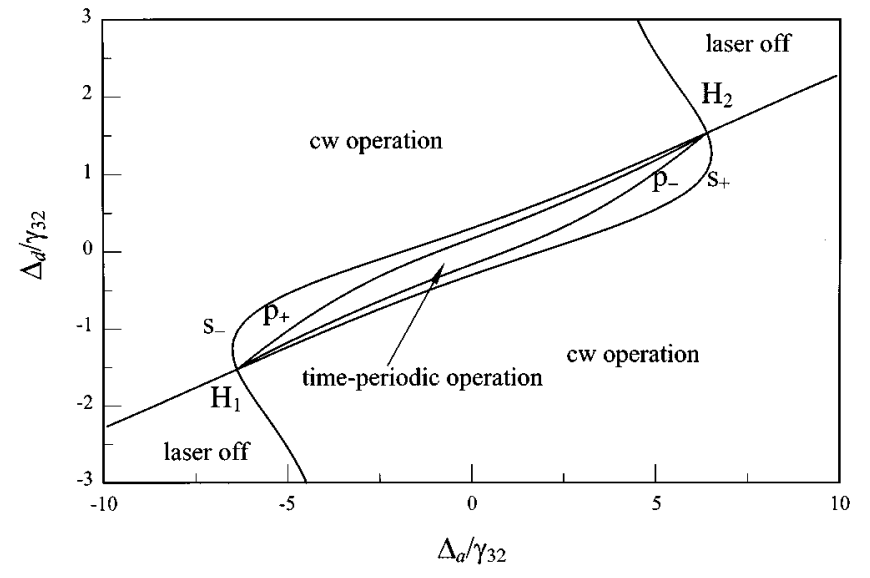

FIG. 6. Bifurcation diagram for Eqs. (1)-(5) and (24). $g=50 \gamma_{32}^{2}, \kappa=10^{-3} \gamma_{32}, \gamma_{31}=\gamma_{32}, r=0.1 \gamma_{32}, \Omega=\gamma_{32}$.

curve $S$ corresponds to the solutions $S_{ \pm}$that have equal intensities in this case. The curve $P$ represent the averaged intensity of the solution with time-periodic output. The averaging was performed over a period of intensity oscillations. The parameter values for Fig. 5(a) [Fig. 5(b)] are the same as for Fig. 4(a) [Fig. 4(b)]. Hence, the solution $P$ is stable (unstable) in Fig. 5(a) [Fig. 5(b)]. Figure 5(c) corresponds to the situation when the codimension-two points $H_{1}$ and $H_{2}$ are of different types. The stable (unstable) self-pulsed solution $P$ bifurcates from the nonlasing solution at the point $H_{1}\left(H_{2}\right)$. Between these points the solutions $P$ and $S_{ \pm}$interchange their stability properties. According to the bifurcation theory there should be some other solutions with time-periodic laser intensity that appear at the points where $P$ and $S_{ \pm}$become unstable. However, since the domain of their existence is very narrow these solutions are not shown.

Figure 6 corresponds to the case where the degenerate Hopf bifurcations labeled by $H_{1}$ and $H_{2}$ take place for nonzero values of the detuning parameters $\left(\Delta_{a}, \Delta_{d} \neq 0\right)$. The solution $S_{+}\left(S_{-}\right)$exists above (below) the curve $s_{+}\left(s_{-}\right)$ and it is stable above (below) the curve $p_{+}\left(p_{-}\right)$. A stable self-pulsed regime exists between the curves $p_{+}$and $p_{-}$. As in Fig. 4(b), the regimes with time-periodic intensity exist only in the narrow strip situated between the codimension- two points $H_{1}$ and $H_{2}$. Note that the fact that the codimension-two points in Fig. 6 occur for $\Delta_{a} \Delta_{d}>0$ agrees with the analytic result obtained for small detuning at the end of Sec. II B 2.

\section{Instability threshold for the case of nonzero cavity detuning}

It follows from Fig. 4 and was already mentioned [14] that in some cases LWI can be more easily achieved in a detuned laser. Let us discuss in more detail the question of whether for given parameter values LWI can appear for any detuning. First consider the case where the cavity eigenfrequency is detuned from resonance $\left(\Delta_{a} \neq 0\right)$ whereas the driving field is on resonance $\left(\Delta_{d}=0\right)$. In this case the necessary and sufficient conditions of sideband instability are given by

$$
\begin{gathered}
g D_{0}-\kappa \gamma_{d}\left(\gamma_{a}^{2}+\gamma_{b}^{2}-2 \Omega^{2}\right)>0 \\
{\left[g D_{0}-\kappa \gamma_{d}\left(\gamma_{a}+\gamma_{b}\right)^{2}\right]^{2}-4 \kappa \gamma_{d}^{2}\left(\gamma_{a}+\gamma_{b}\right)\left(\gamma_{a} \gamma_{b}+\Omega^{2}\right)} \\
\times\left[g s n_{13}+\kappa\left(\gamma_{a}+\gamma_{b}\right)\right]>0
\end{gathered}
$$

where $D_{0}$ is defined by Eq. (15). In particular, in the perfect cavity limit $(\kappa \rightarrow 0)$ the inequalities (26) and (27) are transformed into the single condition $D_{0}>0$, which was obtained for the case when $\Delta_{a}=\Delta_{d}=0$. However, unlike the case of zero detunings, the threshold value of the parameter $g$ defined by the inequalities (26) and (27) tends to zero as $\kappa$ $\rightarrow 0$. Thus, the LWI domain in the laser parameter space can be enlarged by the cavity detuning.

In particular, for the scheme shown in Fig. 1(e) the condition (15) takes the form

$$
r-\frac{\gamma_{31}+\gamma_{32}}{1+\gamma_{32} / 2 \gamma_{a}}>0
$$

Since $\gamma_{a}=\left(\gamma_{31}+\gamma_{32}+r\right) / 2+\gamma_{a}^{n r d}$, it follows from Eq. (28) that the threshold value of the pump parameter increases with increasing $\gamma_{a}^{n r d}$. Let $\kappa=0$ and $\Delta_{a} \rightarrow \infty$. The eigenvalue determining the stability of the nonlasing solution (6) can be written in the form

$$
\lambda_{1}=\frac{g \Omega^{2}}{\Delta_{a}\left[2 \Omega^{2}\left(r+2 \gamma_{31}+2 \gamma_{32}\right)+r \gamma_{32} \gamma_{p}\right]}\left[i\left(\gamma_{31}+\gamma_{32}-r\right)+\frac{r\left(\gamma_{32}+2 \gamma_{a}\right)-2 \gamma_{a}\left(\gamma_{31}+\gamma_{32}\right)}{\Delta_{a}}\right]+O\left(\frac{1}{\Delta_{a}^{3}}\right) .
$$

Therefore, Eq. (28) means that the real part of $\lambda_{1}$ is positive for sufficiently large $\Delta_{a}$. In the radiative limit (5) the inequality (28) coincides with that derived in Ref. [14]:

$$
r^{2}+r \gamma_{32}-\left(\gamma_{31}+\gamma_{32}\right)^{2}>0 \text {. }
$$

\section{The case of off-resonantly driven laser}

Since the situation $\Delta_{d} \neq 0$ is much more complicated to analyze we confine our analytical consideration to the case of the scheme shown in Fig. 1(e) with zero cavity detuning and losses $\left(\Delta_{a}=0, \kappa=0\right)$. For $\Delta_{d} \gg 1$ we get the following expression for the eigenvalue with the largest real part:

$$
\lambda_{1}=\frac{g \Omega^{2}\left[r\left(\gamma_{32}+2 \gamma_{d}\right)-2 \gamma_{d}\left(\gamma_{31}+\gamma_{32}\right)\right]}{\Delta_{d}^{2} r \gamma_{32} \gamma_{d}}+O\left(\frac{1}{\Delta_{d}^{3}}\right) .
$$

For sufficiently large $\Delta_{d}$ this eigenvalue is positive if the inequality

$$
r-\frac{\gamma_{31}+\gamma_{32}}{1+\gamma_{32} / 2 \gamma_{d}}>0
$$



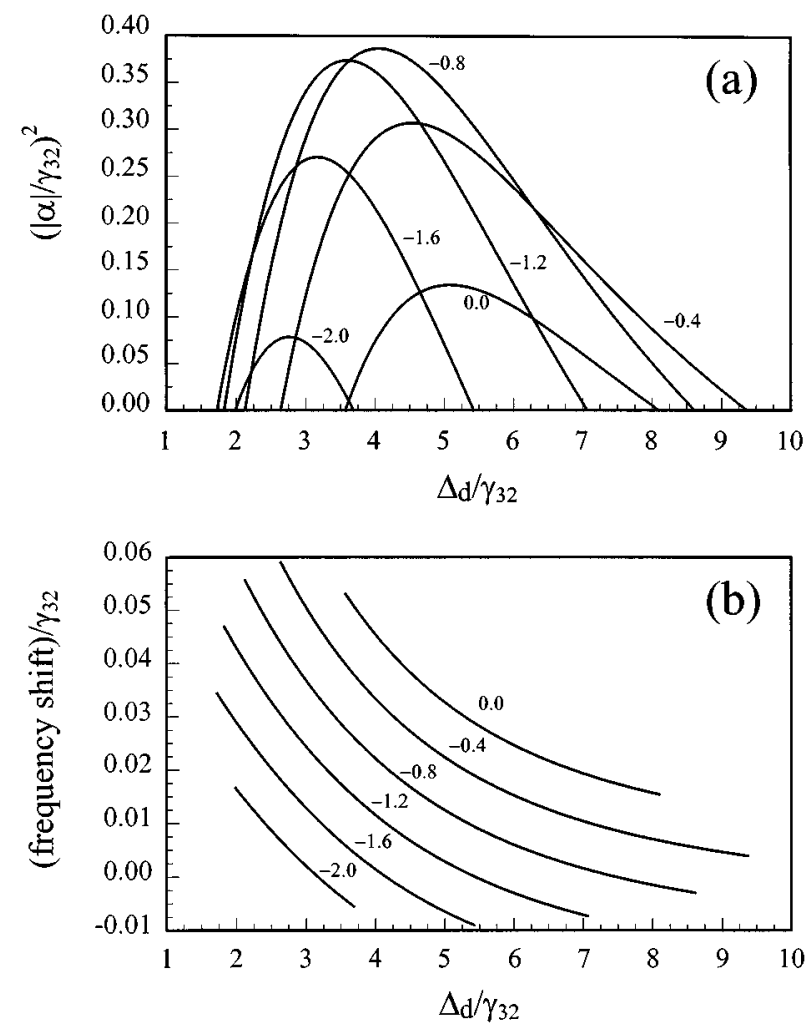

FIG. 7. Dependence of the intensity (a) and the frequency shift (b) of the laser output field on the driving field detuning $\Delta_{d} / \gamma_{32}$ for different values of the cavity detuning $\Delta_{a} / \gamma_{32}$. Calculations were performed with Eqs. (1)-(5) and (24). Parameters are $g=\gamma_{32}^{2}$, $\kappa=0.02 \gamma_{32}, \gamma_{31}=0.2 \gamma_{32}, r=0.75 \gamma_{32}, \Omega=2 \gamma_{32}$. The values of $\Delta_{a} / \gamma_{32}$ corresponding to the various curves are shown in the figure.

is fulfilled. Thus, the condition (29) is sufficient for the instability. In the radiative limit (5) the inequality (29) becomes

$$
r>\gamma_{31}
$$

which coincides with the necessary condition for LWI obtained in [14] for an off-resonantly driven laser. It follows from our consideration that the inequality (29) is the necessary and sufficient condition for the appearance of an instability at some drive detuning $\Delta_{d}$. Note that here, as in Sec. $C$, the instability occurs for arbitrarily small values of the parameter $g$ provided the cavity losses are negligible $(\kappa$ $\rightarrow 0$ ).

In Fig. 7 the results of numerical simulations of Eqs. (1)(5) and (24) with nonzero values of cavity and driving field detuning parameters are presented. The dependence of the laser output intensity on the driving field detuning $\Delta_{d}$ is shown in Fig. 7(a) for different values of the cavity detuning parameter $\Delta_{a}$. This figure corresponds to a situation where LWI is impossible in a resonantly driven laser. However, if the driving field is properly detuned the laser can oscillate on one of the two sidebands. It follows from Fig. 7 that there exist some optimal values of the detuning parameters $\Delta_{d}$ and $\Delta_{a}$ for which the output laser intensity has a maximum. In Fig. 7 these optimal values have opposite signs. This result agrees with the information displayed in Fig. 6 in which the degenerate Hopf bifurcation points occur for $\Delta_{a} \Delta_{d}>0$. Since the line connecting the two Hopf bifurcation points in the
$\left(\Delta_{a}, \Delta_{d}\right)$ plane is situated between the sideband gain maxima, these maxima occur for $\Delta_{a} \Delta_{d}<0$.

Figure 7(b) represents the shift between of the lasing field frequency and the cavity eigenfrequency for the regimes shown in Fig. 7(a).

\section{E. Physical interpretation of the bifurcation diagrams}

In this section, we consider the nonlinear dynamical properties of the laser close to its threshold from an alternative viewpoint. This allows us to illustrate the physical origin of the lasing regimes. We focus on the specific model of a system based on Fig. 1(e). The starting point of our analysis is a Fourier transform of the first equation of system (1)

$$
-i \omega \alpha(\omega)=-\kappa \alpha(\omega)+g x_{31}(\omega) .
$$

In a case of a weak lasing field, the atomic polarization can be expressed in terms of linear susceptibility

$$
g x_{31}(\omega)=i \nu_{0} \chi(\omega) \alpha(\omega),
$$

where $\nu_{0}$ is optical frequency. Assuming $\alpha(\omega) \neq 0$ we can rewrite Eq. (30) as

$$
\begin{aligned}
& \kappa=-\nu_{0} \operatorname{Im} \chi(\omega), \\
& \omega=-\nu_{0} \operatorname{Re} \chi(\omega) .
\end{aligned}
$$

These equations have a form similar to that of the threshold condition and the frequency pulling equation of the usual Lamb semiclassical laser theory. We note, however, that no adiabatic approximation was made in derivating Eqs. (31) and (32). These equations are valid only in the vicinity of the point where the trivial solution becomes unstable and finding their solution (for $g$ and $\omega$ ) is equivalent to carrying out the linear stability analysis of the nonlasing steady-state solution (6).

The complex susceptibility can be calculated from the equations describing the evolution of the density matrix (1). Under the most general conditions it takes the following form (see, e.g., [14]):

$$
\chi(\omega)=-i g \frac{\left(\rho_{33}^{0}-\rho_{11}^{0}\right)+\left(\rho_{11}^{0}-\rho_{22}^{0}\right) \Omega^{2} /\left[\Gamma_{b}(\omega) \Gamma_{d}\right]}{\Gamma_{a}(\omega)+\Omega^{2} / \Gamma_{b}(\omega)},
$$

where $\quad \Gamma_{a}(\omega)=\gamma_{a}-i\left(\Delta_{a}+\omega\right), \quad \Gamma_{b}(\omega)=\gamma_{b}-i\left(\Delta_{a}-\Delta_{d}\right.$ $+\omega), \Gamma_{d}=\gamma_{d}+i \Delta_{d}$, and $\rho_{i i}^{0}$ are the populations of the levels calculated to the zeroth order in probe field. In this expression one can identify two contributions from the atomic coherence [11]. Dynamic Stark shift and splitting are represented by the term proportional to the $\Omega^{2}$ in the denominator of Eq. (33), whereas the term proportional to $\Omega^{2}$ in the numerator reflects the contribution of quantum interference. It is clear that at any given point in a spectrum, the constructive or desctructive character of the latter contribution depends, though not exclusively, on the sign of the population difference $\rho_{11}^{0}-\rho_{22}^{0}$.

Let us now consider several specific cases. First, we focus on the case of resonant drive and lasing fields $\left(\Delta_{a}=\Delta_{d}=0\right)$. Substituting Eq. (33) into the threshold condition (31) shows that the necessary condition for lasing without inversion on the line center $(\omega=0)$ is the absence of inversion on the driven transition $\left(\rho_{11}^{0}>\rho_{22}^{0}\right)$. The reason is if there is an in- 

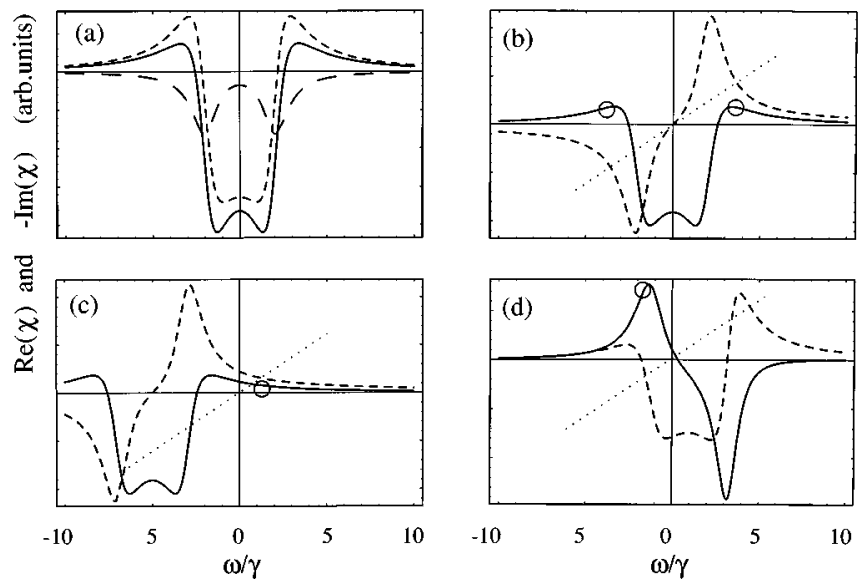

FIG. 8. (a) Contribution to the gain-absorption spectrum from single photon absorption (dashed line) and interference terms (dotted line) separately. The resulting gain is drawn with the solid line. (b) Graphical solution of Eqs. (31) and (32) for the case of perfectly resonant driving field and cavity. Generation occurs on symmetric sidebands at the frequencies marked by circles. (c) Same as (b) but with cavity detuning from atomic resonance. (d) Same as (b) but with driving field detuned from resonance. In both latter cases generation occurs at a single frequency leading to $\mathrm{cw}$ output intensity.

version, $\rho_{11}^{0}<\rho_{22}^{0}$, the contribution of interference to the gain at a line center is destructive, resulting in increased absorption. This is illustrated in Fig. 8(a), where the contributions of the first and second terms of Eq. (33) are plotted separately. The remarkable feature of the interference contribution is that it changes sign for sufficiently large frequencies $|\omega|$. Thus, even though the interference leads to increased absorption on line center, it reduces absorption off resonance and can still result in amplification of the sidebands.

In general, however, amplification is not sufficient for lasing. The reason is that the second oscillation condition (32) is automatically fulfilled for arbitrary coupling only for $\omega=0$. Out of resonance a finite amount of atomic dispersion is always required to compensate the cavity dispersion. It is the reason why the occurrence of a Hopf bifurcation requires a nonvanishing $g$, even in the perfect cavity limit $(\kappa \rightarrow 0)$. This situation is illustrated in Fig. 8(b), where solutions of Eqs. (31) and (32) are found graphically. Clearly, because in the resonant case both equations (31) and (32) are symmetric under the transformation $\omega \rightarrow-\omega$, the sideband solutions appear always in pair with frequencies $\omega= \pm \omega_{0}$ where $\omega_{0}$ is given by Eq. (14). The oscillation conditions for both sidebands are identical. The competition between these sidebands and thus the character of the output intensity are determined by the nonlinear interaction between the sidebands, studied in Sec. III, and depends upon the relation between self- and cross-stauration. Depending on this relation, both stationary and time-periodic output intensities are possible, corresponding to oscillations at either one or both sidebands, respectively.

Let us now turn to the situation of arbitrary cavity detuning $\left(\Delta_{a} \neq 0\right)$ whereas driving field is still resonant $\left(\Delta_{d}=0\right)$. From Eq. (33) it follows that including cavity detunings just shifts the linear susceptibility spectrum by the amount $\Delta_{a}$. In such case, the system (31) and (32) is no longer symmetric with respect to changing the sign of $\omega$. Specifically, the reso- nance condition (32) has to be fulfilled for the two sidebands with unequal gain coefficients [Fig. 8(c)]. Thus, only one of the sidebands crosses the threshold at a time leading to $\mathrm{cw}$ output intensity close to the bifurcation point. One should note at this point that an instability arising in the system in the case of zero cavity detuning $\left(\Delta_{a}=0\right)$ is in a sense artificial. It occurs because of the "improper" tuning of the laser cavity exactly between of two amplification peaks in the gain spectrum. The $\mathrm{cw}$ laser operation is achieved when the laser cavity is tuned to one of the gain peaks rather than to the atomic line center.

Detuning of the driving field can have an even more profound effect on the properties of the three-level amplifiers displaying sideband gain [14]. The resulting susceptibility spectrum is, in general, asymmetric [Fig. 8(d)] and, what is more important, often displays more gain than the corresponding system driven on resonance. Thus, in general, a stable cw solution should emerge from the bifurcation point in this case. The degenerate Hopf bifurcation leading to a time-periodic intensity is, however, possible for certain values of cavity detuning. It occurs for sufficiently small detunings $\Delta_{d}$ when two amplification regions separated by the absorption domain still exist in gain spectrum. In such a case it is possible to find a cavity detuning such that the two sidebands cross the threshold simultaneously. Finally we point out that in the optimal regime of lasing operation, the cavity detuning is in general different from the drive field detuning, and the laser frequency is slightly shifted from the cavity frequency.

\section{CONCLUSION}

We have studied the nonlinear regimes in a three-level ring laser without population inversion in the parameter domain where it exhibits the so-called sideband instability [21]. Very recently it was proposed to use this type of instability to achieve an effective inversionless frequency upconversion in the scheme with driving field applied to a weakly allowed transition [14]. The sideband instability leads to the appearance of a lasing field with a frequency shifted from the line center of the lasing transition even if the cavity and the driving field detunings vanish. For zero detunings the sideband instability was shown to produce a selfpulsed solutions [17]. However, as we have shown, the stability of these solutions and, hence, the problem of their experimental observation cannot be adequately investigated within the framework of the model used in [17]. Here we present a more complete analysis of the self-pulsed regimes in inversionless lasers.

We found that when both sidebands are above the linear threshold, their interaction can lead to different kinds of dynamical behavior. If the self-saturation for the sidebands is stronger than the cross saturation, then they can lock in to produce a stable self-pulsed output. This self-pulsed regime bifurcates from the nonlasing solution only if the instability thresholds are the same for both sidebands. Otherwise, if there is an asymmetry between the sidebands, one of them appears first and gives rise to a cw operation. If the asymmetry is small enough, this regime can undergo a secondary bifurcation leading to a self-pulsed operation.

In the opposite situation of a cross-saturation stronger 
than the self-saturation, the solution with time-periodic lasing intensity is unstable. This means that the sidebands cannot be generated simultaneously and the laser can exhibit only cw output with a frequency shifted from the atomic resonance.

We have derived the normal form equations governing the evolution of the sidebands near the point of the Hopf bifurcation, which is actually a codimension-two degenerate Hopf bifurcation. These equations contain coefficients that depend on the parameters of the original laser equations. We have calculated these coefficients for two different models of the lasing medium. It follows from our calculations that the stability of the self-pulsed regime can be controlled by monitoring the laser parameters. Therefore, depending on the parameter values, either a stable self-pulsed regime or a stable $\mathrm{cw}$ regime bifurcates from the nonlasing solution at the degenerate Hopf bifurcation point. We have also found that under certain conditions the sideband interaction can lead to more complicated periodic and aperiodic regimes which will be described in a subsequent paper.

Even in the limit of zero cavity losses the self-pulsed regimes arise above the threshold $g=g_{0}$ defined by Eq. (13) and they exist only in the narrow strip between the two degenerate Hopf bifurcation points in which both sidebands can be generated simultaneously. On the other hand, LWI in a properly tuned laser with only one exited sideband can be achieved under much more restrictive conditions. Therefore, the presence of a Hopf bifurcation of the nonlasing solution indicates that the LWI threshold can be lowered with the help of detunings. We have shown that the most favorable conditions for LWI can be achieved with only one of the sidebands above the linear threshold. In particular, there exists a parameter domain for which LWI with zero cavity and driving field detunings is impossible, but it can appear in a properly detuned laser on the side of the lasing transition.

We have analyzed analytically and numerically the threshold conditions for the sideband instability for the cases where the cavity and the driving field frequencies are detuned from the corresponding atomic transitions. We have shown that the most favorable conditions for the laser operation in the frequency up-conversion regime are achieved if both cavity and driving field frequencies are shifted from resonance.

\section{ACKNOWLEDGMENTS}

The research in Brussels was supported by the Fonds National de la Recherche Scientifique, the Interuniversity Attraction Pole program of the Belgian government, and a grant from the Services Fédéraux des Affaires Scientifiques, Techniques et Culturelles. The work at Texas A\&M was supported by Office of Naval Research, the Welch Foundation, and the Texas Advanced Technology and Research Program. The collaboration was supported by NATO research Grant No. CRG 960007. S.F.Y. wishes to thank the German Academic Exchange Service for their support through the program HSP II.

\section{APPENDIX: DERIVATION OF THE NORMAL FORM}

Equations (1) can be written in the form

$$
\dot{\mathbf{x}}=L_{1} \mathbf{x}+\mathbf{N}_{1}\left(\mathbf{x}, \mathbf{x}^{*}, \mathbf{y}\right)
$$

$$
\dot{\mathbf{y}}=L_{2} \mathbf{y}+\mathbf{N}_{2}\left(\mathbf{x}, \mathbf{x}^{*}\right)
$$

where the complex vector $\mathbf{x}=\left\{\alpha, x_{31}, x_{32}\right\}^{T}$ and the real vector $\mathbf{y}=\left\{\operatorname{Re}\left(x_{21}-x_{21}^{0}\right), \operatorname{Im}\left(x_{21}-x_{21}^{0}\right), \rho_{11}-\rho_{11}^{0}, \rho_{22}-\rho_{22}^{0}\right\}^{T}$ are both equal to zero for the steady-state solution (12). The nonlinear parts, $\mathbf{N}_{1}$ and $\mathbf{N}_{2}$, contain only second order nonlinearities. At the bifurcation point (13) the matrix $L_{1}$ has two purely imaginary eigenvalues $\lambda_{1}=i \omega_{0}$ and $\lambda_{2}=-i \omega_{0}$. The third eigenvalue of $L_{1}$, and all the eigenvalues of the matrix $L_{2}$, have negative real parts and we will assume that they are well separated from the imaginary axis in the complex plane. In an $\epsilon$ vicinity of the bifurcation point (13) we have $\lambda_{1}=\epsilon(\beta+\delta)+i \omega_{0}$ and $\lambda_{2}=\epsilon(\beta-\delta)-i \omega_{0}$. The quantities $\beta$ and $\delta$ will be evaluated at the end of this Appendix.

We seek the solution of Eqs. (A1), (A2) in the form

$$
\begin{gathered}
\mathbf{x}=\epsilon^{1 / 2}\left[\mathbf{x}_{1}(t, \tau)+\epsilon \mathbf{x}_{3}(t, \tau)+O\left(\epsilon^{2}\right)\right], \\
\mathbf{y}=\epsilon\left[\mathbf{y}_{2}(t, \tau)+O(\epsilon)\right]
\end{gathered}
$$

where $\tau$ is the slow time $\tau=\epsilon t$ [26]. Substituting Eq. (A3) into Eq. (A1) and equating the terms of order $\epsilon^{1 / 2}$ we have the following equation:

$$
d \mathbf{x}_{1} / d t=L_{1}(0) \mathbf{x}_{1},
$$

with the adjoint equation

$$
d \mathbf{x}_{1}^{\dagger} / d t=L_{1}^{T}(0) \mathbf{x}^{\dagger}{ }_{1} .
$$

$L_{1}(0)$ is the matrix $L_{1}$ evaluated at the bifurcation point (13) corresponding to $\epsilon=0$.

Let $\mathbf{V}_{1}\left(\mathbf{V}_{1}^{\dagger}\right)$ be the eigenvector of the matrix $L_{1}(0)$ [transposed matrix $\left.L_{1}^{T}(0)\right]$ corresponding to the eigenvalue $\lambda_{1}=i \omega_{0}$. This vector is normalized as $\left\langle\mathbf{V}_{1}, \mathbf{V}_{1}^{\dagger}\right\rangle=1$. Then the solution of Eq. (A5) has the form

$$
\mathbf{x}_{1}=z_{+}(\tau) \mathbf{V}_{1} \exp \left(i \omega_{0} t\right)+z_{-}(\tau) \mathbf{V}_{1}^{*} \exp \left(-i \omega_{0} t\right)
$$

where $z_{+}(\tau)$ and $z_{-}(\tau)$ are the slowly varying envelopes for the sidebands. Substituting this solution into Eq. (A2) and equating the terms of order $\epsilon$ we get the equation $L_{2}(0) \mathbf{y}_{2}+\mathbf{N}_{2}\left(\mathbf{x}_{1}, \mathbf{x}_{1}^{*}\right)=0$. The solution $\mathbf{y}_{2}$ has terms proportional to $\exp (0 t), \exp \left(2 i \omega_{0} t\right)$, and $\exp \left(-2 i \omega_{0} t\right)$ and it is a second order polynomial in $z_{+}, z_{-}, z_{+}^{*}$, and $z_{-}^{*}$.

Finally, we equate the terms of order $\epsilon^{3 / 2}$. Then we get

$$
\begin{aligned}
L_{1}(0) \mathbf{x}_{3}= & {\left[-d z_{+} / d \tau+(\beta+\delta) z_{+}\right] \mathbf{V}_{1} \exp \left(i \omega_{0} t\right) } \\
& +\left[-d z_{-} / d \tau+(\beta-\delta) z_{-}\right] \mathbf{V}_{1}^{*} \exp \left(-i \omega_{0} t\right) \\
& +\mathbf{N}_{1}\left(\mathbf{x}_{1}, \mathbf{x}_{1}^{*}, \mathbf{y}_{2}\right)
\end{aligned}
$$

$\mathbf{N}_{1}$ includes only third order terms in $z_{+}, z_{-}, z_{+}^{*}$, and $z_{-}^{*}$. The solvability condition for Eq. (A7) requires the orthogonality of its right hand side to the solutions of Eq. (A6). This allows one to avoid in the solution terms that diverge as $t$ $\rightarrow \infty$. Applying the orthogonality conditions we obtain the following equations for the slow envelopes of the sidebands: 


$$
\begin{aligned}
d z_{+} / d \tau= & (\beta+\delta) z_{+}+\int_{0}^{2 \pi / \omega_{0}}\left\langle\mathbf{N}_{1}\left(\mathbf{x}_{1}, \mathbf{x}_{1}^{*}, \mathbf{y}_{2}\right), \mathbf{V}_{1}^{\dagger}\right\rangle \\
& \times \exp \left(-i \omega_{0} t\right) d t \\
d z_{-} / d \tau= & (\beta-\delta) z_{-}+\int_{0}^{2 \pi / \omega_{0}}\left\langle\mathbf{N}_{1}\left(\mathbf{x}_{1}, \mathbf{x}_{1}^{*}, \mathbf{y}_{2}\right), \mathbf{V}_{1}^{\dagger *}\right\rangle \\
& \times \exp \left(i \omega_{0} t\right) d t .
\end{aligned}
$$

These equations can be easily transformed into Eqs. (19), taking into account the symmetry of Eqs. (A1) and (A2) under the transformation $\mathbf{x} \rightarrow \mathbf{x} \exp (i \varphi)$ with abitrary $\varphi$,

The quantities $\epsilon \beta$ and $\epsilon \delta$ are linear in $\Delta_{a}, \Delta_{d}$, and $g-g_{0}$, which describe the small deviations of the bifurcation parameters from the bifurcation point (13). They are given by

$$
\begin{aligned}
\epsilon \beta= & \frac{1}{2 \gamma_{d}\left[\omega_{0}^{2}+\left(\gamma_{a}+\gamma_{b}+\kappa\right)^{2}\right]}\left\{\left(g / g_{0}-1\right) \gamma_{d}\left[\left(\gamma_{a}+\gamma_{b}\right)\left(\Omega^{2}+\left(\gamma_{a}+\kappa\right)\left(\gamma_{b}+\kappa\right)\right)\right]+i \Delta_{a} \gamma_{d}\left[\omega_{0}^{2}+\left(\gamma_{a}+\gamma_{b}+\kappa\right)^{2}+\Omega^{2}\right.\right. \\
& \left.\left.+\left(\gamma_{a}+\kappa\right)\left(\gamma_{b}+\kappa\right)\right]-i \Delta_{d}\left[\left(\gamma_{a}+\kappa\right)\left(\omega_{0}^{2}+\gamma_{d}\left(\gamma_{a}+\gamma_{b}+\kappa\right)+\gamma_{b}\left(\gamma_{b}+\gamma_{d}\right)\right)+\Omega^{2}\left(\gamma_{b}+\gamma_{d}-\kappa\right)\right]\right\}, \\
\epsilon \delta= & \frac{1}{2 \gamma_{d} \omega_{0}\left[\omega_{0}^{2}+\left(\gamma_{a}+\gamma_{b}+\kappa\right)^{2}\right]}\left\{-\Delta_{a} \gamma_{d}\left[\left(\gamma_{a}+\gamma_{b}+\kappa\right)\left(\Omega^{2}+\gamma_{a} \gamma_{b}\right)-\kappa \omega_{0}^{2}\right]+\Delta_{d}\left[( \gamma _ { a } + \gamma _ { b } + \kappa ) \left(\Omega^{2}\left(\gamma_{b}+\gamma_{d}-\kappa\right)+\left(\gamma_{a}+\kappa\right)\right.\right.\right. \\
& \left.\left.\times\left(\omega_{0}^{2}+\gamma_{b}^{2}+\gamma_{b} \gamma_{d}\right)\right)-\omega_{0}^{2} \gamma_{d}\left(\gamma_{a}+\kappa\right)\right]-i\left(g / g_{0}-1\right) \gamma_{d}\left[\left(\gamma_{a}+\gamma_{b}+\kappa\right)\left(\kappa\left(\Omega^{2}+\gamma_{a} \gamma_{b}\right)-\omega_{0}^{2}\left(\gamma_{a}+\gamma_{b}+\kappa\right)\right)\right. \\
& \left.\left.+\omega_{0}^{2}\left(\Omega^{2}+\gamma_{a} \gamma_{b}+\kappa\left(\gamma_{a}+\gamma_{b}\right)-\omega_{0}^{2}\right)\right]\right\},
\end{aligned}
$$

where $g_{0}$ and $\omega_{0}$ are defined by Eqs. (13) and (14), respectively.

[1] M. O. Scully and M. S. Zubairy, Quantum Optics (Cambridge University Press, Cambridge, 1997).

[2] O. Kocharovskaya and Ya. I. Khanin, Pis'ma Zh. Eksp. Teor. Fiz. 48, 581 (1988) [ JETP Lett. 48, 630 (1988)].

[3] S. E. Harris, Phys. Rev. Lett. 62, 1033 (1989).

[4] M. O. Scully, S.-Y. Zhu, and A. Gavrilides, Phys. Rev. Lett. 62, 2813 (1989).

[5] O. Kocharovskaya, Phys. Rep. 219, 175 (1992).

[6] M. O. Scully, Phys. Rep. 219, 191 (1992).

[7] P. Mandel, Contemp. Phys. 34, 235 (1993).

[8] A. Nottelmann, C. Peters, and W. Lange, Phys. Rev. Lett. 70, 1783 (1993).

[9] E. S. Fry, X. Li, D. Nikonov, G. G. Padmabandu, M. O. Scully, A. V. Smith, F. K. Tittel, C. Wang, S. R. Wilkinson, and S.-Y. Zhu, Phys. Rev. Lett. 70, 3235 (1993).

[10] W. E. van der Veer, R. J. J. van Diest, A. Dönszelmann, and H. B. van Linden van den Heuvell, Phys. Rev. Lett. 70, 3243 (1993).

[11] A. S. Zibrov, M. D. Lukin, D. E. Nikonov, L. Hollberg, M. O. Scully, V. L. Velichansky, and H. G. Robinson, Phys. Rev. Lett. 75, 1499 (1995).

[12] G. G. Padmabandu, G. R. Welch, I. N. Shubin, E. S. Fry, D. E. Nikonov, M. D. Lukin, and M. O. Scully, Phys. Rev. Lett. 76, 2053 (1996).

[13] C. Fort, F. S. Cataliotti, T. W. Hänsch, M. Inguscio, and M.
Prevedelli, Opt. Commun. 139, 31 (1997).

[14] S. F. Yelin, M. D. Lukin, M. O. Scully, and P. Mandel (unpublished).

[15] O. Kocharovskaya, R.-D. Li, and P. Mandel, Opt. Commun. 77, 215 (1990).

[16] A. Karawajczyk and J. Zakrzewski, Phys. Rev. A 51, 830 (1995).

[17] V. J. Sánchez-Morcillo, E. Roldán, and G. J. de Valcárcel, Quantum Semiclassic. Opt. 7, 889 (1995).

[18] R. Corbalan, J. Monpart, R. Vilaseca, and E. Arimondo (unpublished).

[19] O. Kocharovskaya and P. Mandel, Opt. Commun. 84, 179 (1991).

[20] O. Kocharovskaya, P. Mandel, and Y. V. Radeonychev, Phys. Rev. A 45, 1997 (1992).

[21] P. Mandel and O. Kocharovskaya, Phys. Rev. A 46, 2700 (1992).

[22] V.I. Arnold, Geometrical Methods in the Theory of Ordinary Differential Equations (Springer, Heidelberg, 1983).

[23] J. Guckenheimer and P. Holmes, Nonlinear Oscillations, Dynamical Systems and Bifurcations of Vector Fields (Springer, Heidelberg, 1983).

[24] J. D. Crawford, Rev. Mod. Phys. 63, 991 (1991).

[25] P. Pepłowski and H. Haken, Phys. Lett. A 120, 138 (1987).

[26] P. Mandel, Theoretical Problems in Cavity Nonlinear Optics (Cambridge University Press, Cambridge, 1997). 